\title{
Philosophie ALS künstlerische Forschung
}

\author{
Philosophy On Stage
}

ARNO BÖHLER

\section{Mythenfreunde, die keine mehr waren}

Auch wenn es für Aristoteles wahr ist, dass »die Menschen zuerst wie jetzt zu philosophieren begonnen hatten«, weil sie über ihr Da-sein in der Welt staunten ${ }^{1}$, so geht er doch davon aus, dass dem Staunen der griechischen Dichter und Mythenfreunde noch der rechte wissenschaftliche Sinn abging. Zwar gibt er uns zu bedenken, dass auch sie bis zu einem gewissen Grad Philosophen sind, »setzt sich doch ein Mythos aus Wunderbarem zusammen «2 ${ }^{2}$ aber die Art und Weise, wie sie sich über die Welt wundern und die kosmische Stellung des Menschen inmitten der Natur im Rekurs auf tradierte Mythen poetisch erklären, vermochte die Philosophen unter den Griechen schon damals nicht mehr wirklich zu überzeugen. Dass ein Sophist wie Gorgias die Dichtung als eine Art Zauberei und Magie betrachtete ${ }^{3}$ und Platon die Dichter bekanntlich aus seinem Idealstaat verbannte, zeigt nur, dass die Freunde der Weisheit im öffentlichen Leben der griechischen Polis in der Regel durch eine skeptische Haltung zur überlieferten Wahrheit des Mythos auffielen ${ }^{4}$. Sogar Platon, der Philosoph unter den Philosophen der abendländischen Philosophie, der »als Dichter unter den großen Denkern gilt und in der Tat seine Philosophie mit ei-

1 Aristoteles: Metaphysik, Stuttgart: Reclam 1984, 982b.

2 Ebd.

3 Hermann Diels/Walther Kranz (Hg.): Die Fragmente der Vorsokratiker, Hildesheim: Weidmann 2004, 82 B 11, 23.

4 Vgl. Helmut Heit: Der Ursprungsmythos der Vernunft. Zur Genealogie der griechischen Philosophie als Abgrenzung vom Mythos, Dissertation Universität Hannover 2003, S. 205-229; Burkert, Walter: Hermes Bd. 88, Wiesbaden: Franz Steiner Verlag 1960, S. 159-160. 
nem künstlerischen Anspruch verbindet $\aleph^{5}$, gerade er war derjenige, der in der Dichtkunst einen der schärfsten Rivalen der Philosophie identifizierte. ${ }^{6}$ Vor allem lehnte er die Herrschaft der Dichter innerhalb der Stadtmauern ab, weil sie für Platon schlechte Volkserzieher sind, die falsche Vorstellungen über die Götter und das sittliche Leben unter dem Volk verbreiten und durch ihre Reden und Schriften eher die Begierden und Leidenschaften fördern, als den klaren Verstand. Oft zitieren die antiken Philosophen das Sprichwort »Vieles lügen die Sänger « ${ }^{7}$ gerade so, als würden sie sich durch die Opposition zu den Dichtern selbst erst definieren. Also qua Negation.

Bis zu einem bestimmten Grad gehört es seither zum guten Ton innerhalb der akademischen Disziplin der Philosophie, dass das, was Philosophen tun, nicht mit dem verwechselt werden darf, was Dichter tun. - - - Wer künftig an einer platonischen Akademie Gehör finden will, wird das Hören auf den Logos ${ }^{8}$ dem Hören auf den Mythos im Gefolge Platons vorziehen müssen und nur noch jenen künstlerischen Praxen einen philosophischen Wert zubilligen dürfen, die dem Ideal der Förderung eines sittlich guten Lebens innerhalb des philosophischen Regimes der Vernunft förderlich sind. Philosophie und Kunst treten damit in einen Besorgnis erregenden Zwiespalt zueinander, der die Geschichte der Philosophie von Platon bis Hegel - und darüber hinaus - maßgeblich mitbestimmen wird.

Erst Nietzsche hat den Abgrund dieser Kluft zwischen Philosophie als künstlerischer bzw. wissenschaftlicher Praxis als kardinales Problem der zeitgenössischen Philosophie wieder entdeckt, wenn er etwa im Rückblick auf seinen eigenen philosophischen Werdegang im Frühjahr-Sommer i888 resümiert: »Über das Verhältnis von Kunst und Wahrheit bin ich am frühesten ernst geworden: und noch jetzt stehe ich mit einem heiligen Entsetzen vor diesem Zwiespalt. «9 Wie ich im Folgenden zeigen möchte, ist dieser Zwiespalt noch immer ein ungelöstes Problem der zeitgenössischen Philosophie.

5 Otfried Höffe: Aristoteles, München: C.H. Beck 2006, S. 69.

6 Vgl. Platon: Sämtliche Werke, Reinbek bei Hamburg: Rowohlt 1994, Politeia II-III, X; Sophistes 235eff.; Nomoi VII 816d-817e.

7 Aristoteles: Metaphysik, 983a.

8 Vgl. Diels/Kranz: Fragmente der Vorsokratiker, Heraklit 22 B 50.

9 Nietzsche, Friedrich: Nachgelassene Fragmente, Frühjahr-Sommer 1888, KSA 13, Berlin/ New York: Walter de Gruyter 1980, S. 500. 


\section{Philosophie als Prinzipienwissenschaft}

Für Aristoteles, jenen Philosophen, der neben Platon über viele Jahrhunderte der abendländischen Philosophiegeschichte als der Philosoph der akademischen Welt schlechthin gehandelt wurde, beruht der rangmäßige Vorrang der Philosophie gegenüber mythisch-poetischen Erklärungsversuchen vor allem in der wissenschaftlichen Methode, die die griechischen Philosophen seit dem 6. Jhd. v. Chr. zu entwickeln begannen. Für ihn waren sie nämlich nicht nur chronologisch, sondern auch rangmäßig die Ersten, die, in einem emphatischen Sinne, gelernt hatten, prinzipiell zu denken, indem sie die Vielfalt empirischer Einzelerscheinungen auf ihnen inhärente allgemeine Prinzipien ( $\alpha \rho \chi \alpha \grave{\imath})$

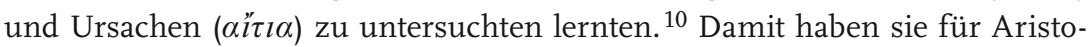
teles Epochales geleistet; wurde damit doch erstmals die angemessene Methode einer wissenschaftlich-epistemischen Untersuchung der Welt entdeckt und zur philosophischen, anstatt dichterisch-mythischen Welterklärung herangezogen.

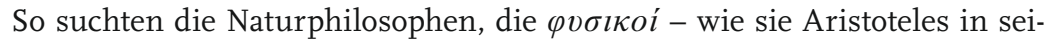
ner Physik nennt ${ }^{11}$ - kraft der Annahme eines Urstoffes nach einem ersten unveränderlichen Prinzip, aus dessen Veränderungen sie die Entstehung des Alls zu ergründen und zu begründen versuchten. ${ }^{12}$ Auch wenn dieser Versuch für Aristoteles inhaltlich in die Irre ging, so haben sie damit doch die rechte Fragestellung einer jeden künftigen philosophischen Weltbetrachtung ans Licht befördert. Im Anschluss an diese Bestimmung des Denkens wird akademisch Philosophieren fortan für viele Jahrhunderte der europäischen Geistesgeschichte heißen, die Frage nach dem Grund der ursächlichen Entstehung von etwas zu stellen. In einem wörtlich zu verstehenden Sinne wird Philosophie nach Aristoteles damit zur Grundlagenforschung (»Archéologie«); zu einer Fundamentalwissenschaft, der es um die systematische Erkenntnis der Gründe geht, die für das Entstehen und Vergehen konkreter Einzelerscheinungen operativ verantwortlich zeichnen. ${ }^{13}$ Und zwar so, dass diese Gründe fortan auf so-

10 Vgl. Aristoteles: Physik, Hamburg: Meiner Verlag 1987, 184a 1; Aristoteles: Metaphysik, $981 \mathrm{~b}-$ 982a.

11 Aristoteles: Physik, 184a 2.

12 Vgl. Arno Böhler: »TheatReales Raumdenken«, in: Thomas Erne/Peter Schüz (Hg.), Die Religion des Raumes und die Räumlichkeit der Religion, Göttingen: Vandenhoeck \& Ruprecht 2010, S. 35-51.

13 Vgl. Wolfgang Wieland: Die aristotelische Physik, Göttingen: Vandenhoeck \& Ruprecht 1962 , S. 63-65. 
kratische Art und Weise selbst argumentativ bewiesen werden müssen, indem sie unter Experten intersubjektiv diskutiert und in Hinblick auf ihre sachliche Überzeugungskraft auf ihre Konsistenz hin dialektisch verhandelt werden.

Im Unterschied zu den in Griechenland auftretenden Philosophen blieb den Dichtern und Mythenfreunden jener Zeit diese wissenschaftliche Denkungsart offenkundig noch fremd und der Weg zur philosophischen Erkundung der Welt qua Prinzipienforschung daher methodisch auch verschlossen. - - - Was unter anderem daran liegen mochte, dass ihre poetische Art der Verwunderung über die Welt noch mit dem affektiven Ergriffenwerden von der Welt einherging, so dass sie die Welt aus ihren Stimmungen heraus dichterisch deuteten, anstatt sie auf ihre inhärente Ursächlichkeit hin philosophisch zu ergründen. Die sokratische Nüchternheit, die das wissenschaftliche Gemüt eines Philosophen idealerweise charakterisiert, blieb ihren leidenschaftlich-trunkenen Gemütern fremd; - und damit auch die nötige intellektuelle Distanz zu den Dingen, die wissenschaftliches Forschen auszeichnet; - - - wenn nicht sogar erotisiert. Denn Sokrates, so Nietzsche in seiner Götzen-Dämmerung, »war auch ein großer Erotiker«. ${ }^{14}$ Übte seine Nüchternheit auf die alten Eliten Athens doch eine fast magische Anziehungskraft aus, die sie durch und durch faszinierte, wenn nicht sogar mehr ...

»Aber Sokrates errieth noch mehr. Er sah hinter seine vornehmen Athener; er begriff, dass sein Fall, seine Idiosynkrasie von Fall bereits kein Ausnahmefall war. Die gleiche Art von Degenerescenz bereitete sich überall im Stillen vor: Das alte Athen gieng zu Ende. - Und Sokrates verstand, dass alle Welt ihn nöthig hatte, — sein Mittel, seine Kur, seinen Personal-Kunstgriff der Selbst-Erhaltung ... Überall waren die Instinkte in Anarchie; überall war man fünf Schritte weit vom Excess: das monstrum in animo war die allgemeine Gefahr. >Die Triebe wollen den Tyrannen machen; man muss einen Gegentyrannen erfinden, der stärker ist $\ldots . .{ }^{15}$

14 Friedrich Nietzsche: Götzen-Dämmerung, KSA 6, Berlin/New York: Walter de Gruyter 1980, S. 71.

15 Ebd. 


\section{Der Ruf nach Sokrates}

Platons Symposion ist vielleicht gerade darum ein so wunderbar paradoxes Meisterwerk, weil es den Konflikt zwischen Philosophie als einer wissenschaftlich-sokratischen Praxis und Philosophie als einer künstlerisch-dramatischen Inszenierung sowohl inhaltlich, als auch formal ein für alle Mal auf unnachahmliche Art und Weise ins Werk gesetzt hat. ${ }^{16}$

Wenn Alkibiades trunken in die hochkarätig besetzte Expertenrunde angesehener Redner hereinplatzt, die sich im Symposion zusammengefunden hatten, um Eros zu preisen, dann führt uns Platon in dieser spektakulären Szene auf dramatische Art und Weise vor Augen, was es heißt, an dem mythisch-dichterischen Wesensbegriff von Eros festzuhalten, der eher an Dionysos, als an einen Philosophen gemahnt. Ihm, dem vom Weingott trunkenen Alkibiades, stellt Platon Sokrates, den Inbegriff eines nüchtern-trunkenen Philosophen entgegen, um anhand dieser beiden Figuren idealtypisch zu demonstrieren, wie sich die alte, dionysische Lobrede von Eros zu der neuen sokratischen verhält.

Ein gravierender Unterschied zwischen diesen beiden Weisen, das Wesen einer Sache zu rühmen, besteht für Platon in der unterschiedlichen Gemütsverfassung, in der sich diese beiden Helden im Symposion befinden. Besessen von der alten mythischen Ansicht der antiken Dichter und Feldherrn, geht Alkibiades offenkundig immer noch davon aus, dass man von dem, worüber man spricht, tatsächlich leibhaftig ergriffen und affektiv heimgesucht werden muss, wenn man das Wesen einer Sache wirklich angemessen begreifen möchte. Ein Redner, der über Eros spricht, vermag dies in diesem Fall nur dann angemessen zu tun, wenn und solange er von Eros selbst (erotisch) stimuliert wird; sich der Redner also selbst in einer trunkenen Stimmung befindet, aus der heraus er Eros auf erotisierte und erotisierende Weise huldigt. Der leiblich-affektive Zustand, in dem sich Alkibiades befindet, während er »über« Eros redet, ist für ihn offenkundig also selbst noch ein konstitutives Merkmal der »Definition «dessen, was es zu be-greifen gilt, wenn man das »wesen « (verbal) von Eros wirklich begreifen will.

Wer im alten mythisch-dichterischen Sinne über Eros sprechen möchte, muss ihn am eigenen Leib also selbst erfahren. So wie der Vortrag der alten Rhapsoden über die tradierten Mythen selbst in hymnisch-liedhafter Art und Weise »kunstvoll« vorgetragen, sprich gesungen wurden, so glaubt auch Alki-

16 Vgl. Martin Puchner: The Drama of Ideas, Oxford: Oxford University Press 2010; Freddie Rokem: Philosophers and Thespians, Stanford: Stanford University Press 2010. 
biades Eros im vorsokratischen Sinne dichterisch-trunken preisen zu müssen, um ihn angemessen zu ehren, anstatt ihn philosophisch-nüchtern zu denken.

Ganz anders verhält es sich mit der Art und Weise, wie Sokrates selbst Eros rühmt. Sein Vortragsstil zeichnet sich genau dadurch aus, dass er durch seine Nüchternheit zu faszinieren weiß. Nicht die Trunkenheit, sondern die sachhaltige Argumentation wirkt in seinem Fall auf die anwesenden Redner erotisierend. Dass er als Philosoph von Eros gerade nicht affiziert wird, während er über ihn spricht, zeichnet Sokrates vor allen anderen Rednern aus. Geht es ihm doch nicht mehr um die Artikulation seines eigenen leibhaftigen Begehrens, sondern um das Zur-Sprache-bringen der Idee des Schönen schlechthin, die dafür verantwortlich zeichnet, dass uns überhaupt etwas Schönes widerfahren kann, das uns begehrlich erscheint.

Um dieses ideelle Wesen des Eros in den Blick zu bekommen, bedarf es einer merkwürdigen platonischen Umwendung unserer Forschungsperspektive: weg von der eigenen leiblich-empirischen Erfahrung, hin zur begrifflichargumentativen Verhandlung der Idee von Eros schlechthin, durch deren Dialektik die Idee der Schönheit an sich zum Vorschein gebracht werden soll und im günstigsten Fall gedanklich auch zum Vorschein gebracht wird. Die animierende Wirkung von Eros auf unser eigenes Gemüt, in der er sich in uns selbst leibhaftig-affektiv bekundet, gehört für Philosophen vom Schlage eines Sokrates seither nicht mehr zum eidetischen Wesensbegriff der Sache selbst. Wer das Wesen von Eros künftig philosophierend, d. h. in nüchtern-sokratischer Manier objektiv-wissenschaftlich, und nicht mehr mythisch-dichterisch erfassen möchte, muss dies fortan auf diskursive Art und Weise im Medium der Sprache tun. Und zwar im Zuge der logischen Erörterung der eidetischen, ansich-seienden Wesensbestimmungen, die Eros begrifflich zukommen. Nicht die Physis - d. h. das leiblich-affektive Anwesen von Eros selbst inmitten der Natur -, sondern der Logos - d. h. die diskursive Verhandlung seines Wesens im Medium Sprache - ist fortan das angemessene Mittel, in dem die konstitutiven Wesensmerkmale des Begriffs Eros sprachlich entborgen und folglich ideellgeistig zum Sich-zeigen gebracht werden.

Seit Platon ist es konsequenterweise nicht mehr das verbale „wesen“ einer Sache, das Philosophen in der Regel interessiert, sondern das substantivierte Wesen ihrer rein begrifflich zu erfassenden Idee. Selbst der Vollzug von Tätigkeiten wie »gehen«, »reden«, »laufen«, »lachen«, »schreiben« etc. werden durch den grammatischen Trick platonisierender Denker, Verbalsubstantive von Verben zu bilden, fortan rein formal-begriffliche Wesenheiten, die wir logisch-formal bestimmen können, ohne die betreffende Tätigkeit de facto selbst durchführen, 
also tätigen zu müssen. Wir sprechen dann in sokratisch-nüchterner Manier formal über »das Gehen«, »das Laufen«, »das Lachen«, »das Schreiben«, »das Sehen«, ohne in der Tat gehen, laufen, lachen, schreiben, sehen zu müssen, während wir über diese Tätigkeiten reden. Nicht mehr der aktuelle Vollzug des Gehens selbst, sondern die formal-logische Struktur von Gehen überhaupt ist von nun an das, was sokratisch gesinnte Philosophen und Wissenschaftler interessiert.

Eine Denkungsart, die vor allem Tänzern und Schauspielern höchst fremd sein muss, wenn sie vor dem konkreten Problem stehen, in actu auf eine bestimmte Art und Weise über eine Bühne gehen zu müssen. Und zwar mit dem Teil des ihnen de facto dafür zur Verfügung stehenden »Fleisches der Welt « ${ }^{17}$, das es ihnen in der Tat ermöglicht, im Rekurs auf ihre Leiblichkeit diesen Vollzug auf diese eine, oder aber jene andere Art und Weise konkret durchzuführen. Während eine Künstlerin also vor dem Problem steht, über aktuelle Leibvollzüge nachdenken zu müssen, wenn ihr ihr »Gehen«, »Reden«, »Laufen« etc. zum Problem wird, kann der sokratische Philosoph die Idee des Gehens auch im Liegen oder Stehen, in welcher Lage auch immer, ermitteln. Und zwar darum, weil der leibliche Vollzug des Gehens für ihn keine zusätzliche Wesensinformation darüber liefert, was es prinzipiell betrachtet denn überhaupt heißt, zu gehen. Nicht nur (sic!) in einem konkreten Fall, sondern schlechthin allgemein.

Eine sokratische Methode des Denkens, die etwa beim Atmen bald an die Grenze kommt. Muss doch auch Sokrates in actu atmen, wenn er über die Atmung spricht, und zwar so, dass er diesen konkreten Vollzug beim Nachdenken über die Idee des Atmens implizit immer schon faktisch vollziehen muss, wenn er über die Idee der Atmung schlechthin nachdenken möchte. Jeder Versuch, dem konkreten Vollzug des Atmens die Reflexion über ihr eidetisches Wesen vorzuziehen, wird in diesem Fall über kurz oder lang scheitern. (Auch das substantivierte Wesen des Menschen oder gar das An-sich-sein eines Körpers wird seit Sokrates nicht mehr im Zuge des physisch-leiblichen Anwesens eines Menschen oder eines lokal auftretenden Körpers inmitten der »Natur« [Physis] gedacht, sondern im Zuge der diskursiven Verhandlung der betreffenden Ideen innerhalb der Sprache. Der leibliche Zugang zum [verbalen] „wesen“ einer uns angehenden Sache beginnt sich in seinem Gefolge philosophiegeschichtlich

17 Maurice Merleau-Ponty: Das Sichtbare und das Unsichtbare, München: Wilhelm Fink Verlag 1986, S. $181-182$. 
immer mehr zu verschließen. Das Reden über eine Sache beginnt das physische Anwesen der Sache selbst schließlich gänzlich zu ersetzen. Der linguistic turn in der Philosophie des 20. Jhdts. bekommt von daher gesehen ein bedrohliches Gesicht.)

Offenkundig heißt akademisch philosophieren nach Sokrates so viel wie, sich von der »wesens«-Macht einer Sache (verbal) affektiv so zu distanzieren lernen, dass wir sie unabhängig von unserer leiblichen Gestimmtheit rein rational zu betrachten und diskursiv zu verhandeln vermögen. Aus Angst, als einem leiblichen Zustand des Selbstgewahrens, wird dadurch in guter alter idealistischer Manier die Angst schlechthin. Aus dem leibhaftigen Anwesen eines Menschen der Mensch an sich. Aus der leiblichen Erfahrung von Lust ein an-sich-seiendes geistiges Gebilde namens Lust, das mit der von uns selbst leiblich-empfundenen Lust nichts mehr, oder doch nur wenig, gemein hat. Nicht mehr das Sehen von Farben, sondern die diskursive Beschreibung derselben, die auch ein Farbenblinder verstehen kann, macht jetzt den originär gegebenen (phänomenalen) Gegenstand einer wissenschaftlichen Betrachtung solcher Phänomene aus.

Gerade das, was lebensweltlich naiv als die eigentlich »hymnische « Präsenz von Eros gelesen wird, das Wecken von erotischen Gefühlen, ist im Anschluss an die platonische Erfindung der Begriffsperson Sokrates zu einem parasitären, akzidentiellen Moment einer bloß noch ästhetisch-sinnlichen, aber noch nicht logisch-begrifflichen Bestimmung seines Wesen geworden. Der Weg hin zur Reterritorialisierung der antiken Philosophie im Deutschen Idealismus ist damit vorgebahnt ${ }^{18}$ - und folglich die alte Festschreibung einer Diskriminierung leiblich-affektiver Zustände zugunsten feststehender logischer Wesensbestimmungen. Selten demonstriert ein Wissenschaftler heute noch das Gehen leiblich, wenn er es wissenschaftlich beschreiben, und nicht bloß »kunstvoll« zeigen möchte.

Diese sokratische Disziplinierung der akademischen Philosophie ist inzwischen soweit fortgeschritten, dass in der Regel jeder, der heute eine wissenschaftliche Disziplin erlernt, völlig ungefragt in diese Art und Weise zu denken hineinsozialisiert wird. Ganz unabhängig davon, ob jemand in Europa, Japan, Afrika oder sonst wo auf akademische Art und Weise philosophischwissenschaftlich diszipliniert wird. Inzwischen handelt es sich dabei um eine weltweit standardisierte universitäre Tatsache, die Nietzsche wohl als erster

18 Vgl. Gilles Deleuze: Was ist Philosophie?, Frankfurt a.M.: Suhrkamp 1996, S. 108-127. 
namhafter Philosoph Europas nicht mehr nur zu rühmen, sondern auch kritisch zu hinterfragen wusste. Könnte es doch sein, dass die sokratische Befreiungsgeschichte des wissenschaftlichen Geistes, durch ihre über zweitausend Jahre andauernde Geschichte inzwischen einen kritischen Punkt erreicht haben könnte, in dem diese Geschichte selbst eine neue Form von Bedrohung angenommen hat, die zumindest einige Philosophen unserer Zeit in der Nachfolge Nietzsches höchst bedenklich stimmt. Denn:

»Wenn man nöthig hat, aus der Vernunft einen Tyrannen zu machen, wie Sokrates es that, so muss die Gefahr nicht klein sein, dass etwas Anderes den Tyrannen macht. Die Vernünftigkeit wurde damals errathen als Retterin, es stand weder Sokrates, noch seinen >Kranken< frei, vernünftig zu sein, - es war de rigueur, es war ihr letztes Mittel. Der Fanatismus, mit dem sich das ganze griechische Nachdenken auf die Vernünftigkeit wirft, verräth eine Nothlage: Man war in Gefahr, man hatte nur Eine Wahl: entweder zu Grunde zu gehen oder - absurd vernünftig zu sein. «19

Für post-sokratische Philosophen wie Nietzsche haben sich die Zeichen der Zeit inzwischen radikal geändert. Für sie sind es nicht mehr die Dichter, die das sokratische Philosophieren gefährden, sondern Sokrates selbst; — insofern unsere Zeit, im Anschluss an sein Bild von Denken, inzwischen eben global Gefahr läuft, »absurd vernünftig« zu werden.

\section{Post-sokratisches Philosophieren}

Vor dem Hintergrund dieser gewandelten historischen Matrix unternimmt Philosophy On Stage ${ }^{20}$ den Versuch, den künstlerischen Anspruch philosophischer Praktiken wieder in die akademische Disziplin der Philosophie aufzunehmen und in die wissenschaftliche Forschungspraxis von Philosoph_innen methodisch einzuarbeiten. Da es sich dabei um ein neues philosophisches Forschungsformat handelt, das sich selbst nicht länger in klassisch-sokratischer Manier in Opposition zu den Künsten definiert, wird Philosophie im

19 Friedrich Nietzsche: Götzen-Dämmerung, Berlin/New York: Walter de Gruyter 1980, S. 72.

20 Mediathek Philosophy On Stage\#3, http://homepage.univie.ac.at/arno.boehler/php/?p=2018 vom 1. 7. 2013. 
Zuge dieser post-sokratischen Revision der Philosophie selbst zu einer Form künstlerisch-wissenschaftlichen Forschens (art-based-research).

Da die klassische Geschichte der europäischen Philosophie, wie eingangs skizziert, vom Ausschluss der Künste aus der Philosophie lebt, um sich selbst gerade als alternative wissenschaftliche Methode in Abgrenzung zu künstlerischen Praktiken zu etablieren und zu definieren, kann das neue Suchformat Philosophy On Stage logischerweise auf kein historisch vorgegebenes Beispiel von Philosophie rekurrieren, auf das es sich berufen könnte, wenn es den Ausschluss künstlerischer Praktiken in der Philosophie nicht länger wiederholen möchte. Zwar gibt es unzählige Beispiele innerhalb des europäischen Kanons der abendländischen Philosophie, in dem wir auf Beispiele stoßen, in denen Philosoph_innen ihre Wissenschaft selbst mit einem künstlerischen Anspruch verbunden haben. Platon wurde schon genannt, Nietzsche wäre wohl das bekannteste Beispiel der neueren Philosophiegeschichte. Gleichwohl handelt es sich dabei nur um marginale Randerscheinungen innerhalb der Übermacht der sokratischen Norm des Philosophierens, deren Form die akademische Welt heute noch beherrscht, so dass der ästhetische Anspruch an das Philosophieren selbst aus den ehrwürdigen Hallen der akademischen Philosophie inzwischen fast gänzlich verschwunden ist. Philosophie ist eine Wissenschaft und keine Kunst. Mit dieser weit verbreiteten Ansicht hat nicht nur der Chor antiker Philosophen sein Standesbewusstsein in Abhebung zu den Dichtern generiert; auch heute noch herrscht darüber ein weit verbreiteter Konsens unter akademischen Philosoph_innen.

\section{Die Ereignishaftigkeit des Denkens denken}

Aufgrund dieser historisch gewordenen Lage bewegen wir uns bei unserem Versuch, Philosophie selbst als Form künstlerischen Forschens zu verstehen, in der Tat in einem Gebiet philosophischer Grundlagenforschung, das es uns konsequenterweise nicht mehr erlaubt, bei der Bestimmung dessen, was es heißt, zu philosophieren, einfach auf den herrschenden sokratischen Begriff von Philosophie zurückzugreifen. Diesen mystischen Grund der (sokratischen) Autorität ${ }^{21}$ müssen wir vielmehr grundlegend dekonstruieren, um uns den Weg

21 Vgl. Jacques Derrida: Gesetzeskraft. Der mystische Grund der Autorität, Frankfurt a.M.: Suhrkamp 1991. 
zu einem post-sokratischen Verständnis von Philosophie zu bahnen, das eher an Nietzsche, als an Platon mahnt. So wie Platon, vor vielen Jahrhunderten, die Figur von Sokrates erst dichten musste, um die Herrschaft der Dichter und Mythenfreunde in Athen zu brechen, so müssen wir, im Durchgang durch die Dekonstruktion der sokratischen Figur des Denkens, uns den Begriff einer post-sokratischen Philosophie erst erschaffen, um das sokratische Bild des Denkens, das uns heute beherrscht, zu brechen.

Ein post-sokratisches Forschungsformat wie Philosophy On Stage forscht daher, um eine berühmte Formulierung Kants aufzunehmen, »ohne Begriff «22, weil es sich einen adäquaten Begriff von dem, was es tut, wenn es philosophiert, im Laufe seiner research performance konstruktiv erst erschaffen wird müssen. Steht die Demonstration einer Philosophie, die sich selbst als künstlerisch-wissenschaftliche Praxis zu vollziehen beginnt, historisch gesehen doch noch aus.

Ganz im Sinne von Jean-François Lyotards Beantwortung der Frage, was ist postmodern? - befindet sich ein post-sokratischer Philosoph daher in derselben Lage wie ein Künstler.

»Der Text, den er schreibt, das Werk, das er schafft, sind grundsätzlich nicht durch bereits feststehende Regeln geleitet und können nicht nach Maßgabe eines bestimmenden Urteils beurteilt werden, indem auf einen Text oder auf ein Werk nur bekannte Kategorien angewandt würden. Diese Regeln und Kategorien sind vielmehr das, was der Text oder das Werk suchten. Künstler und Schriftsteller arbeiten also ohne Regeln; sie arbeiten, um die Regeln dessen zu erstellen, was gemacht worden sein wird. Daher rührt, dass Werk und Titel den Charakter eines Ereignisses haben. Daher rührt auch, dass sie für ihren Autor immer zu spät kommen, oder, was auf dasselbe führt, dass die Arbeit an ihnen immer zu früh beginnt. Postmodern wäre also als das Paradox der Vorzukunft (post-modo) zu denken.“ 23

22 Immanuel Kant: Kritik der Urteilskraft, Stuttgart: Reclam 1991, S. 93.

23 Jean-François Lyotard: „Beantwortung der Frage: Was ist postmodern?», in: Peter Engelmann (Hg.): Postmoderne und Dekonstruktion. Texte französischer Philosophen der Gegenwart, Stuttgart: Reclam 1990, hier S. $47 f$. 
Wenn Philosophy On Stage ${ }^{24}$ den Anspruch stellt, Philosophie wieder als künstlerisch-wissenschaftliche Forschungspraxis verstehen zu lernen, in der es nicht nur um die begriffslogische Verhandlung von Sprache, sondern auch um das verbale, ereignishafte Geschehen des leiblich-affektiven Erscheinens eidetischer Wesensstrukturen geht, dann reiht sich dieses Bild des Denkens offenkundig in ein Denken des Ereignisses ein, das nicht nur viele aktuelle Denkströmungen innerhalb der Philosophie ${ }^{25}$, sondern auch wichtige zeitgenössische Ästhetiken ${ }^{26}$ charakterisiert. Für eine solche Denkungsart ist charakteristisch, dass der empirische, situativ-datierbare Kontext, in dem sich eine Sache zeigt, für die Sache selbst kein rein äußerliches Moment mehr bildet. ${ }^{27}$ Vielmehr gehört der leibliche Kontext, in dem sich etwas zeigt, in guter alter vorsokratischer Manier, von nun an wieder mit zur »post-sokratischen Definition« des Eidos einer Sache.

Im Anschluss an Sybille Krämer können wir in diesem Zusammenhang von einer korporalisierenden Konzeption von Performanz sprechen. Diese ist »inspiriert von der Erfahrung der künstlerischen >Performanceく, von einer Entwicklungstendenz in den Künsten, die oftmals als Übergang vom >Werk zum Ereignis< charakterisiert wird. « ${ }^{28}$ In einer solchen performativen Form des Philosophierens spielen die Orte, Räume und Gegenstände, innerhalb der sich ein Gedanke zeigt, eine mit entscheidende Rolle für das, was er bedeutet und ob er sich in einem bestimmten territorialen Milieu überhaupt zeigen kann und zeigen darf. »Das Physische im Vollzug einer Aufführung - der Körper des Schauspielers und alle sinnlichen sichtbaren Attribute einer Aufführung - bleiben dabei nicht länger Zeichen für einen dahinter liegenden immateriellen Sinn,

24 TRP12-G21, 2010-2013, Generating Bodies, research project sponsored by the Austrian Science Fund (FWF): http://homepage.univie.ac.at/arno.boehler/php/?p=3361 vom 1. 7. 2013.

25 Vgl. Marc Rölli: Ereignis auf Französisch. Von Bergson bis Deleuze, München: Wilhelm Fink 2004; Arno Böhler/Susanne Granzer: Ereignis Denken. TheatRealität, Performanz, Ereignis, Wien: Passagen Verlag 2009; Susanne Valerie [Granzer]: Schauspieler außer sich. Exponiertheit und performative Kunst, Bielefeld: transcript 2011.

26 Vgl. Dieter Mersch: Ereignis und Aura, Frankfurt a.M.: Suhrkamp 2002.

27 Vgl. Krämer, Sybille (Hg.): Performanz und Medialität, München: Wilhelm Fink 2004; Sybille Krämer/Ekkehard König (Hg.): Gibt es eine Sprache hinter dem Sprechen?, Frankfurt a.M.: Suhrkamp 2002.

28 Krämer (Hg.): Performanz und Medialität, S. 17; vgl. Mersch: Ereignis und Aura, S. 163. 
der in der Materialität des Darstellungsgeschehens lediglich zur Erscheinung kommt.«29

In einer Art Umkehrung der platonischen Ideenlehre bilden die sinnlichen Aufführungsbedingungen im Kontext eines solchen post-sokratischen Bilds vom Denken gleichsam das räumliche Milieu, das die territoriale Emanation bestimmter Sinnstrukturen ermöglicht oder eben auch nicht ermöglicht; - und zwar kraft der Anordnung der das Territorium gemeinsam »populierenden« Körper, kraft der sie es für eine bestimmte Zeit miteinander teilen, lokal bewohnen und damit auch physisch auf vielfache Art und Weise perspektivieren. Die Frage, wie bestimmte territoriale Milieus kreiert und so in Szene gesetzt werden können, dass sie die Emanation bestimmter »Ideen« territorial ermöglichen, wird jetzt ein konstitutives Problem einer jeden so gearteten philosophischen Forschungspraxis.

Vom 24. bis 27. November 20II hat das Philosophie \& Performance Festival Philosophy On Stage $\#^{30}$ das klassische, durch unzählige Wiederholungen immer wieder bestärkte sokratische Bild von Philosophie herausgefordert, indem es das von Ludwig Wittgenstein maßgeblich selbst konzipierte Palais Wittgenstein im Dritten Wiener Gemeindebezirk zu einem Territorium erklärt hat, das der Anbahnung eines solchen neuen, post-sokratischen Formats des Philosophierens ein territoriales Zuhause gewähren wollte, in dem 64 Künstler_innen, Philosoph_innen und Wissenschaftler_innen daran gebaut haben, das Denken der Philosophie als Modus künstlerischen Forschens demonstrieren zu lernen. ${ }^{31}$

$29 \operatorname{Krämer}(\mathrm{Hg}$.): Performanz und Medialität, S. 18.

30 Philosophy On Stage \#3 (2011): http://homepage.univie.ac.at/arno.boehler/php/?p=3142 vom vom 1. 7. 2013; vgl. auch Philosophy On Stage \#1 (2005): http://www.univie.ac.at/ performanz/index.php/home/ vom 1. 7. 2013; Philosophy On Stage \#2 (2007): http:// www.univie.ac.at/performanz/pos2/ vom 1. 7. 2013.

31 Mediathek Philosophy On Stage \#3, http://homepage.univie.ac.at/arno.boehler/php/?p=2018 vom 1. 7. 2013. 


\section{Bibliographie}

Aristoteles: Metaphysik, Stuttgart: Reclam I984

Aristoteles: Physik, Hamburg: Meiner Verlag I987

Böhler, Arno/Granzer Susanne: Ereignis Denken. TheatRealität, Performanz, Ereignis, Wien: Passagen Verlag 2009

Böhler, Arno: »TheatReales Raumdenken«, in: Thomas Erne/Peter Schüz (Hg.),

Die Religion des Raumes und die Räumlichkeit der Religion, Göttingen: Vandenhoeck \& Ruprecht 20IO, S. 35-5I

Burkert, Walter: Hermes Bd. 88, Wiesbaden: Franz Steiner Verlag 1960

Deleuze, Gilles: Was ist Philosophie?, Frankfurt a.M.: Suhrkamp I996

Derrida, Jacques: Gesetzeskraft. Der »mystische Grund der Autoritä««, Frankfurt a.M.: Suhrkamp I99I

Diels, Hermann/Kranz, Walther (Hg.): Die Fragmente der Vorsokratiker, Hildesheim: Weidmann 2004

Hegel, Georg Wilhelm Friedrich: Vorlesungen über die Geschichte der Philosophie I, Frankfurt a.M.: Suhrkamp I97I

Hegel, Georg Wilhelm Friedrich: Vorlesungen über die Geschichte der Philosophie. Griechische Philosophie I. Thales bis Kyniker, Hamburg: Meiner Verlag 1989

Heit, Helmut: Der Ursprungsmythos der Vernunft. Zur Genealogie der griechischen Philosophie als Abgrenzung vom Mythos, Dissertation Universität Hannover 2003

Höffe, Otfried: Aristoteles, München: C.H. Beck 2006

Kant, Immanuel: Kritik der Urteilskraft, Stuttgart: Reclam I99I

Krämer, Sybille/König, Ekkehard (Hg.): Gibt es eine Sprache hinter dem Sprechen?, Frankfurt a.M.: Suhrkamp 2002.

Krämer, Sybille (Hg.): Performanz und Medialität, München: Wilhelm Fink Verlag 2004

Lyotard, Jean-François: »Beantwortung der Frage: Was ist postmodern?«, in: Peter Engelmann (Hg.): Postmoderne und Dekonstruktion. Texte französischer Philosophen der Gegenwart, Stuttgart: Reclam I990, S. 33-48

Marx, Karl: Karl Marx - Friedrich Engels - Werke, Berlin: Dietz I968

Mersch, Dieter: Ereignis und Aura, Frankfurt a.M.: Suhrkamp 2002

Mersch, Dieter: Posthermeneutik, Berlin: Akademie Verlag 20 оо

Merleau-Ponty, Maurice: Das Sichtbare und das Unsichtbare, München: Wilhelm Fink Verlag 1986 
Mediathek Philosophy On Stage \#3, http://homepage.univie.ac.at/arno.boehler/ php/?p=20I8 vom I. 7. 2013

Nietzsche, Friedrich: Götzen-Dämmerung, KSA 6, Berlin/New York: Walter de Gruyter ig80

Nietzsche, Friedrich: Nachgelassene Fragmente, Frühjahr-Sommer I888, KSA I3, Berlin/New York: Walter de Gruyter I980

Philosophie Performance: http://performancephilosophy.ning.com/ vom I. 7 . 2013

Philosophy On Stage \#I (2005): http://www.univie.ac.at/performanz/index. php/home/ vom I. 7. 2013

Philosophy On Stage \#2 (2007): http://www.univie.ac.at/performanz/pos2/ vom I. 7. 2013

Philosophy On Stage \#3 (20II): http://homepage.univie.ac.at/arno.boehler/ $\mathrm{php} / \mathrm{p}=3 \mathrm{I} 42$ vom I. 7.2013

Platon: Sämtliche Werke, Reinbek bei Hamburg: Rowohlt 1994

Puchner, Martin: The Drama of Ideas, Oxford: Oxford University Press 2010

Rokem, Freddie: Philosophers and Thespians, Stanford: Stanford University Press 2010

Rölli, Marc: Ereignis auf Französisch. Von Bergson bis Deleuze, München: Wilhelm Fink 2004

TRPI2-G2I, 2010-20I3, Generating Bodies, research project sponsored by the Austrian Science Fund (FWF): http://homepage.univie.ac.at/arno.boehler/ $\mathrm{php} /$ ?p $=336$ I vom I. 7. 2013

Valerie [Granzer], Susanne: Schauspieler außer sich. Exponiertheit und performative Kunst, Bielefeld: transcript 2oII

Wieland, Wolfgang: Die aristotelische Physik, Göttingen: Vandenhoeck \& Ruprecht I962 